\title{
FORMATION ANALYSIS OF MULTI-FREQUENCY SIGNALS OF LASER INFORMATION MEASURING SYSTEM
}

\author{
Sergey Herasimov \\ Department of Arms Air Defense Ground Forces ${ }^{1}$ \\ gsvnr@ukr.net \\ Oleksandr Tymochko \\ Department of Navigation and Motion Control ${ }^{1}$ \\ timochko.alex@gmail.com \\ Oleksii Kolomiitsev \\ Department of Automobile and Armored Vehicles \\ Military Institute of Tank Troops National Technical University Kharkiv Polytechnic Institute" \\ 192 Poltavs'kyy Shlyakh str., Kharkiv, Ukraine, 61000 \\ alexus_k@ukr.net \\ Gennadiy Aloshin \\ Department of Transport Communications \\ Ukrainian State Academy of Railway Transport \\ 7 Feuerbach sq., Kharkiv, Ukraine, 61050 \\ dr.aloshin@mail.ru \\ Oleksandr Kriukov \\ Department of Missile Artillery Weapons ${ }^{2}$ \\ National Academy of National Guard of Ukraine \\ 3 Zakhysnykiv Ukrainy sq., Kharkiv, Ukraine, 61001 \\ kam14@ukr.net \\ Oleksandr Morozov ${ }^{2}$ \\ maa-ngu@ukr.net \\ Volodymyr Aleksiyev \\ Department of Cyber Security and Information Technology \\ Simon Kuznets Kharkiv National University of Economics \\ 9-A Nauky ave., Kharkiv, Ukraine, 61166 \\ aleksiyev@gmail.com \\ ${ }^{1}$ Ivan Kozhedub Kharkiv National Air Force University \\ 77/79 Sums'ka str., Kharkiv, Ukraine, 61023 \\ ${ }^{2}$ National Academy of the National Guard of Ukraine \\ 3 Zakhysnykiv Ukrainy sq., Kharkiv, Ukraine, 61001
}

\footnotetext{
Abstract

Improving the accuracy of measuring motion parameters and the volume of information exchange with aircraft requires the modern development of multifunctional radio systems that allow the combination of information and measuring channels due to frequency (time) signal selection. However, radio systems have limitations on the number and accuracy of the measurement of aircraft motion parameters, as well as information transfer rates. These restrictions will make it possible to remove laser systems for measuring with high accuracy the parameters of motion of aircraft. Similar laser systems are widely used in transmitting information to stationary or quasi-stationary objects. As a result, there was a need to overcome the contradiction between the requirements for the technical characteristics of radio systems and the capabilities of the existing scientific and methodological apparatus for synthesizing the signals of laser information-measuring systems for monitoring the parameters of aircraft motion. The theoretical foundations of the formation and selection of laser signals for combining the information and measuring channels of laser information-measuring
} 
systems are proposed and investigated. A comparison is made with known methods of combining (combining) the information and measuring channels of radio engineering systems and the features of using a spectrum of laser radiation formed by pairs of longitudinal modes that "color" each channel signal for their selection at reception. When receiving optical pairs of longitudinal modes, the intermode beat frequencies turn into radio engineering subcarriers that are well filtered and selectable. Based on the results of mathematical modeling and combinations of longitudinal modes, the choice of elements of a modified selector of longitudinal modes of laser information-measuring systems is substantiated. This determines the importance and usefulness of work for applied systems for measuring the parameters of movement of aircraft.

Keywords: aircraft, laser information-measuring system, laser radiation spectrum, intermode beat frequency.

DOI: $10.21303 / 2461-4262.2019 .00984$

\section{Introduction}

Laser information measuring systems (LIMS) due to the fact that the photodetector converts the amplitude modulation of the radio signal into a video signal, have either a wide range of information signals, or any spectrum of the parameter is measured. Therefore, due to the bad compatibility and filtering of signals, there is an interdependence between these channels $[1,2]$.

In radio engineering systems (RES), the definition and control of motion parameters (MP) of aircraft, the number of transmitters and receivers of measuring signals depends on the number of such MP [3-7]. The vast majority of existing RESs measure four main MPs: range, speed, altitude and azimuth. In this case, in the RES useful signal is amplified, distributed over the channels and filtered in its passband (PB). This procedure is not possible for LIMS due to the conversion of the optical signal into a video signal [8-12]. Then, laser systems (LS) with isolated signals can be of high quality.

Realization of high coherence of laser radiation (LR) does not require a wide PB, but it requires overcoming other difficulties in application - targeting LR on aircrafts and tracking them in parameters.

The multichannel information-measuring systems for controlling aircraft MP, which operate in the radio-technical wave range, have been sufficiently studied [3-7, 13-15]. The problems of combining (and separating) channels in such systems are solved for mobile radio lines mainly by the frequency method of channel separation [3, 10, 11], and for cable lines using digital time-division multiplexing methods [14]. The disadvantages of such systems are the limitation on the speed of information transmission, low accuracy of measuring aircraft MP due to the significant speed of the latter.

In the leading countries of the world, they focus on the creation of combined (multifunctional) systems that solve several problems [15-18]. In such systems, the high accuracy of measuring aircraft MP is ensured through the use of a laser radiation source, and its search and tracking due to the wide-angle television channel. In this case, two joint LSs are used: transmitting and receiving. The disadvantage of such systems is the need to coordinate the joint work of the LS components, leading to its technical complications and an increase in the cost of development and further operation.

The advantages and principles of constructing optoelectronic sensing systems for moving and stationary objects using LR are well studied [16, 19, 20]. It is shown that the use of the tuning of pulsed-periodic radiation can increase the information capacity of the system and expand the detection and recognition of various moving and stationary objects. However, the issues of identifying and recognizing various high-speed moving objects, which include aircraft, are not considered.

A review of the information indicates that at present the scientific and methodological apparatus for generating and selecting laser signals for combining information and measuring channels has not received adequate coverage. Therefore, it is advisable to further develop the theoretical foundations for the formation of laser multi-frequency signals for connecting LIMS channels using a single radiation source (laser) and a single laser transmitting and receiving part of the system.

The aim of research is in development of proposals for the formation of multi-frequency LIMS signals.

To achieve the aim, the following objectives are set:

- determine the features of the formation of laser multi-frequency signals of the laser information-measuring system; 
- substantiate the list of initial data for the synthesis of measuring signals of laser information transmission systems;

- offer an analytical basis for calculating the structure of signals at the output of a laser information-measuring system;

- describe the operation of the modified longitudinal mode selector of the laser information-measuring system;

- carry out practical testing of the calculation of the channel characteristics of the modified longitudinal mode selector of the laser information-measuring system.

\section{Proposals for the development of a laser information-measuring system}

2. 1. Formation of a list of source data for the synthesis of measuring signals of laser transmission systems

Laser radiation is multimode radiation, that is, a combination of longitudinal and transverse modes. In this case, the longitudinal modes are found at different distances relative to each other. This circumstance leads to a decrease in the LR power and, consequently, to a decrease in the range of the system, to a decrease in the number and accuracy of the measured aircraft MP. However, on the other hand, in the nodes of each mode the number of atomic inversions is less than in antinodes; therefore, the mode-modulation contributes to compaction of the inverse layers during injection and to an increase in the radiation in the laser. Using synchronization methods for the longitudinal modes of a single-mode multi-frequency LR (Fig. 1) allows one to achieve high characteristics of the initial radiation of each mode. This opens up the possibility of improving the work due to the frequency selection of each pair of longitudinal modes, or the frequency of intermode beat $[1,5,15]$.

So, LR allow to create a transmitting part of LIMS with combined information and measuring channels. The system will provide high-precision measurement of the inclined range to the aircraft, azimuth and elevation angles, radial and tangential (angular) speeds, and will also provide stable angular automatic tracking and transmission of control commands to the aircraft and the like.

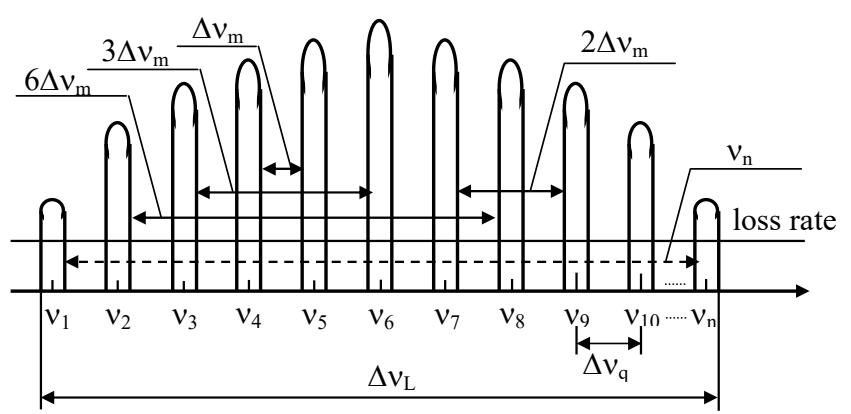

Fig. 1. The spectrum of a single-mode multi-frequency LR with synchronization of longitudinal modes, which is used in LIMS

Due to the oncoming scan of the pairs of partial LR radiation pattern (RP) in each of the two orthogonal planes on the receiving side of the system, the following is possible (Fig. 2):

- generate error signals along two coordinate axes and determine with high accuracy the azimuth and elevation angles taking into account the sign of the angle of deviation of the aircraft from the equal-signal direction (ESD). To do this, measure the shift of the periods of the envelope bursts of pulses from the intermode beat frequencies during one full pass of the LR RP in the forward and reverse scanning directions. Next, measure the shift of similar periods of the envelopes in the opposite direction in each of the two orthogonal planes. Next, a comparison is made of the difference between the shifts of the periods. The time-pulse measurement method is used;

- determine the angular velocity of the aircraft, compensate for the components of speed and acceleration in the corners in the channel of the angular automatic tracking of the aircraft in the direction. To do this, measure the duration of the half-periods of the envelope bursts of pulses from the frequencies of intermode beats in one pass of the LR RP in one direction of scanning. Next, a 
similar length of the half-cycle of envelopes in the opposite direction in each of the two orthogonal planes is measured. Find the difference of such durations by the time-pulse measurement method;

- with high accuracy to determine the radial speed of the aircraft by the Doppler effect and the slant range by the signal delay.

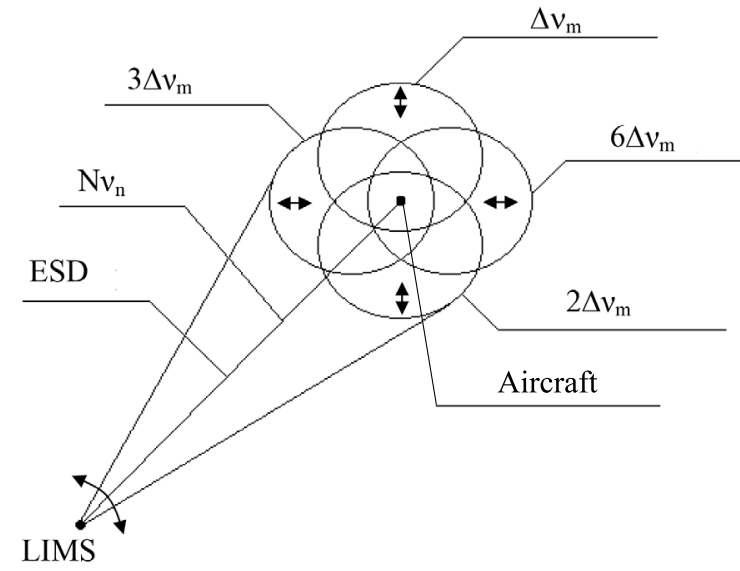

Fig. 2. Scanning 4 LR RP in orthogonal planes and creation of ESD in aircraft

The most important application of acoustic-optical modulators (AOMs) in the LIMS construction is the control of the laser generation mode in the transmitting part of the system to obtain the synchronization mode of longitudinal modes by actively synchronizing them. This mode can be implemented as follows. Laser generation begins on one central mode, and then, as a result of intermode interaction, the generation is also excited on other modes with the necessary phase difference, as a result of which all modes become rigidly coupled.

Therefore, in order to synchronize the longitudinal modes and tightly stabilize the intermode beat frequencies of the LR spectrum, it is proposed to use an active AOM, and as a YAG: $\mathrm{Nd}^{3+}$ active element, a laser (an aluminum-yttrium garnet crystal with an admixture of trivalent neodymium ions) is proposed.

Known LSs (information and measurement) that emit a signal on an optical carrier using photo detectors (PD) turn an optical signal into a video signal. For information systems, on the one hand, this is useful, because the wider the joint venture, the greater the speed of information transfer, the more language channels in the time division of channels.

However, on the other hand, it is not effective enough. So, with a wide PB on an optical carrier, there are more noise photons in comparison with signal photons that act in a narrow SP. In this case, the signal-to-noise ratio is worse. Signal amplification and filtering are also required. But, positive is the lack of resonant amplifiers. The disadvantages are also associated with the fact that the PB of existing optical modulators and demodulators is limited and amounts to approximately $1 \mathrm{GHz}$. This means that the PB of the laser of the transmitter is not undermounted, and wave compaction is possible only at carrier frequencies. In addition, angular modulation and demodulation at the optical carrier frequency is practically impossible.

For measuring channels of a system in which a narrow spectrum of the signal is needed, a small PB, which is poorly filtered when a video signal is used. To overcome such drawbacks of laser transmission lines, a combination of each LIMS channel with frequency selection is proposed in order to select its own pair of longitudinal modes of equal level, which should not be repeated with their different composition.

Each pair of longitudinal modes, falling on one PD, causes intermode beating at its frequencies falling into the radio engineering wave range. These frequencies are amplified, converted with the frequency of the radio receiver and filtered even in a very narrow PB. At the same time, LIMS receives in addition all the advantages of the radio engineering range. Intermode beats are significantly spaced in frequency, which is important for channel selection in frequency. Angular modulation also becomes possible if one of a pair of carrier longitudinal modes is modulated. 
The formation of the total LR RP and the creation of an equal-signal direction is associated with stringent requirements on the emission spectrum of a single-mode multi-frequency laser transmitter, that is, high-precision synchronization of longitudinal modes and stabilization of the frequencies of intermode beats.

2. 2. Analytical expressions for the calculation of the signal structure at the output of the laser information-measuring system

Let's consider the structure of the signals probing at the output of the LIMS.

The optical oscillations of the electric field of each of the frequencies of the selected pair of modes can be written in the following form:

- the first pair of modes $\Delta v_{54}=v_{5}-v_{4}=\Delta v_{m}$ :

$$
\begin{aligned}
& E_{5}(t)=A_{5} \sin \left[2 \pi\left(v_{L}+5 \Delta v_{m}\right) t+\phi_{5}\right] \\
& E_{4}(t)=A_{4} \sin \left[2 \pi\left(v_{\mathrm{L}}+4 \Delta v_{\mathrm{m}}\right) t+\phi_{4}\right],
\end{aligned}
$$

where $A$ is the amplitude of the mode; $v_{L}$ is the laser frequency; $\Delta v_{m}$ is the intermode beat frequency; $t$ is the time; $\varphi$ is the mode phase;

- the second pair $\Delta v_{97}=v_{9}-v_{7}=2 \Delta v_{m}$ :

$$
\begin{aligned}
& E_{9}(t)=A_{9} \sin \left[2 \pi\left(v_{L}+9 \Delta v_{m}\right) t+\phi_{9}\right] \\
& E_{7}(t)=A_{7} \sin \left[2 \pi\left(v_{L}+7 \Delta v_{m}\right) t+\phi_{7}\right]
\end{aligned}
$$

- the third pair $\Delta v_{63}=v_{6}-v_{3}=3 \Delta v_{m}$ :

$$
\begin{aligned}
& E_{6}(t)=A_{6} \sin \left[2 \pi\left(v_{L}+6 \Delta v_{m}\right) t+\phi_{6}\right] \\
& E_{3}(t)=A_{3} \sin \left[2 \pi\left(v_{L}+3 \Delta v_{m}\right) t+\phi_{3}\right]
\end{aligned}
$$

- the fourth pair $\Delta v_{82}=v_{8}-v_{2}=6 \Delta v_{m}$ :

$$
\begin{aligned}
& E_{8}(t)=A_{8} \sin \left[2 \pi\left(v_{L}+8 \Delta v_{m}\right) t+\phi_{8}\right] \\
& E_{2}(t)=A_{2} \sin \left[2 \pi\left(v_{L}+2 \Delta v_{m}\right) t+\phi_{2}\right] .
\end{aligned}
$$

The result of the summation of electromagnetic waves at the PD input for the first pair of frequencies is determined by the formula:

$$
\begin{gathered}
E_{5}(t)+E_{4}(t)=2 A_{54} \sin \frac{2 \pi\left(v_{L}+5 \Delta v_{m}\right) t+\phi_{5}+2 \pi\left(v_{L}+4 \Delta v_{m}\right) t+\phi_{4}}{2} \times \\
\times \cos \frac{2 \pi\left(v_{L}+5 \Delta v_{m}\right) t+\phi_{5}-2 \pi\left(v_{L}+4 \Delta v_{m}\right) t-\phi_{4}}{2} .
\end{gathered}
$$

During photodetection, the first factor disappears, since the optical carrier turns into a photocurrent with the amplitude of the second factor with a difference frequency of intermode beating. At the PD output, the electric fields of each intermode beat frequency are already in the radio engineering range and the result can be written in the form of the following analytical expressions:

$$
\begin{gathered}
E_{54}(t)=A_{9} \sin \left[2 \pi\left(\Delta \mathrm{v}_{m}\right) t+\phi_{9}\right], \\
E_{97}(t)=A_{16} \sin \left[2 \pi\left(2 \Delta v_{m}\right) t+\phi_{16}\right],
\end{gathered}
$$




$$
\begin{aligned}
& E_{63}(t)=A_{9} \sin \left[2 \pi\left(3 \Delta v_{m}\right) t+\phi_{9}\right] \\
& E_{82}(t)=A_{10} \sin \left[2 \pi\left(6 \Delta v_{m}\right) t+\phi_{10}\right] .
\end{aligned}
$$

The selection of the necessary longitudinal modes and combinations of their pairs is possible due to the use of the proposed modified longitudinal mode selector (MLMS).

\section{3. Description of the operation of the modified longitudinal mode selector of the laser information-measuring system \\ MLMS is based on narrow-band Fabry-Perot interferometers (FPI), the number of which is equal to the number of longitudinal modes and their selected combinations (Fig. 3).}

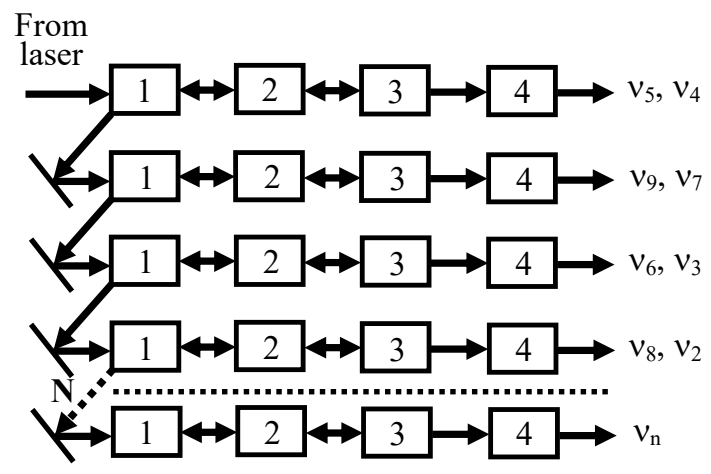

Fig. 3. MLMS functional diagram

MLMS contains in each of the first 4 channels (Fig. 3):

- optical radiation polarizer (1);

- passive phase plate $\lambda / 4$, which returns the vector $E$ of the outgoing radiation at an angle of $45^{\circ}$ in one pass (2);

- narrowband FPI, which is tuned to a signal of a certain combination of modes (frequencies) (3); quency pair);

- optical quantum amplifier (4), for amplification of the output radiation (selected fre-

- auxiliary mirrors designed for LR canalization.

After the optical polarizer of the selected frequency pair $v_{8}, v_{2}$, the last $\mathrm{N}$ channels are connected in series, each of which is composed of:

- optical polarizer of radiation, passive phase plate;

- narrow-band FPI tuned to a signal of one specific mode (frequency) $v_{1} \ldots v_{n}$;

- an optical quantum amplifier for amplifying output radiation.

The following assumptions are used to quantify the magnitude of losses in each optical channel of the MLMS. Losses due to inaccurate communication of the plane of polarization of the polarizing prism and the incoming LR, as well as radiation, twice passed the quarter-wave phase plate, taking into account the assumption of phase shift, is estimated according to the Malus' law:

$$
I=I_{0} \cdot \cos ^{2} \Delta \alpha
$$

where $\Delta \alpha$ is the angle between the plane of polarization of the prism and LR.

An analysis of expression (10) indicates the insignificance of the corresponding losses.

Thus, the magnitude of these losses does not exceed $3 \cdot 10^{-4}$. Moreover, the phase shift tolerance of a modern phase plate is from 0.5 to 1 degree, and the accuracy of measuring the rotation of the plane of polarization is two orders of magnitude higher. 


\section{Analysis of channel characteristics of the modified longitudinal mode selector of a laser information-measuring system}

Since a linearly polarized LR is used, it is possible to ensure its angle of incidence on the edge of the elements of the MLMS channels is equal to the Brewster angle, at which reflection is practically absent. Therefore, the reflection loss from the faces of the elements of the MLMS channels can be ignored.

Due to the use of high quality materials in the manufacture of prisms, the extinction coefficient does not exceed $10^{-5}$. Therefore, the loss of drugs caused by its weakening in the optical elements of the MLMS can be ignored.

LR losses in the sewer mirror can’t be taken into account, since modern multilayer dielectric mirrors have a reflection coefficient $(0.95 \ldots 0.98)$. In addition, they can be replaced by prisms with full internal reflection, in which there are practically no losses.

To estimate the loss of LR in an FPI, an expression is used that describes the dependence of the transmission of an ideal FPI on the difference in the path of interference rays when illuminated with monochrome radiation with a wavelength $\lambda$ :

$$
T(\gamma)=\frac{(1-R)^{2}}{1+R^{2}-2 R \cdot \cos (2 \pi \cdot \gamma)} \cdot T_{0},
$$

where $R=\sqrt{r_{1} \cdot r_{2}}$, and $r_{1}, r_{2}$ are the reflection coefficients of each of the FPI mirrors;

$$
\gamma=2 t / \lambda-E(2 t / \lambda)
$$

is share of the interference order; $E(2 t / \lambda)$ is a symbol of the whole share; $2 t$ is the optical path difference;

$$
T_{0}=[(1-R-\varepsilon) /(1-R)]^{2}
$$

is the absolute value of the maximum transmittance (when the difference in the optical path is a multiple of half $\lambda$ ).

Fig. 4 shows plots of the dependences of the absolute value of the maximum transmittance on the reflection coefficient for absorption values of dielectric mirrors $\varepsilon=0.003 ; 0.005 ; 0.007 ; 0.01$ respectively.

A dependency analysis indicates that the maximum transmittance at absorption of dielectric mirrors $T_{0}$ for all values is more than 0.9 at $R \leq 0.8$. Moreover, for $\varepsilon=0.005$, changes occur in $T_{0}=0.81$ at $R=0.95$ and to $T_{0}=0.94$ at $R=0.75$. For $\varepsilon=0.01$, changes occur in $T_{0}=0.64$ at $R=0.95$ and to $T_{0}=0.92$ at $R=0.75$.

The minimum transmittance when the optical path difference is a multiple of the fourth part $\lambda$ is described by the expression:

$$
T_{\min }=\left(\frac{1-R}{1+R}\right)^{2} \cdot T_{0} .
$$

From expression (12), the dependences of the minimum transmittance $T_{\min }$ on the reflection coefficient are calculated for various values of the absolute value of the maximum transmittance $T_{0}$, the graphs of which are shown in Fig. 5.

From an analysis of the corresponding dependences (Fig. 5), it is possible to conclude with respect to the values of the minimum transmittance $T_{\min }$, which ranges from $T_{\min }=1.7 \cdot 10^{-2}$ at $\mathrm{R}=0.75$ to $T_{\min }=1.7 \cdot 10^{-5}$ at $\mathrm{R}=0.95$.

The dependence of the relative transmission is obtained:

$$
\frac{T(\gamma)}{T_{0}}=J(\gamma)=\frac{(1-R)^{2}}{1+R^{2}-2 R \cdot \cos (2 \pi \gamma)},
$$


according to the results of mathematical modeling for one MLMS channel a graph is completed, which is an Airy function (Fig. 6).

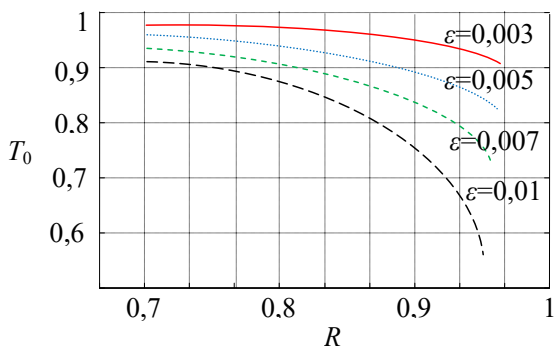

Fig. 4. Graphs of the dependences of the absolute value of the maximum transmittance $T_{0}$ on the reflection coefficient

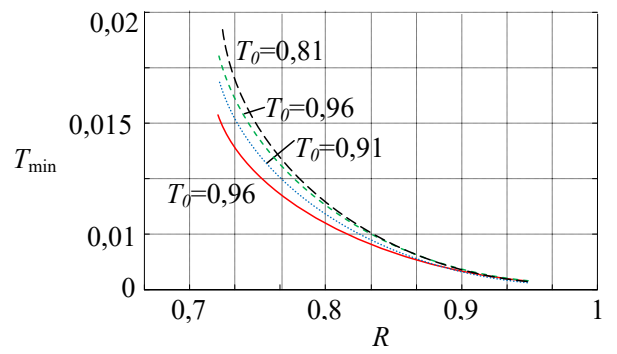

Fig. 5. Graphs of the dependences of the minimum transmittance $T_{\min }$ on the reflection coefficient for the absolute maximum transmittance

From Fig. 6 it is clear that the numerical value of the channel transmission $n$ for each LR mode varies between $T_{0}$ and $T_{\min }$, depending on what value the Airy function acquires for $\gamma_{n, m}$ for the FPI in this channel:

$$
T_{n, m}=T_{0} \cdot J\left(\gamma_{n, m}\right)
$$

For LIMS information channels, the intensity of the output LR is:

$$
\begin{aligned}
I_{i+4, i} \approx T_{0} \cdot\left[1-T_{0} \cdot J\left(\gamma_{i+3, i}\right)\right] \cdots\left[1-T_{0} \cdot J\left(\gamma_{4, i}\right)\right]\left[1-T_{0} \cdot J\left(\gamma_{3, i}\right)\right] \times \\
\times\left[1-T_{0} \cdot J\left(\gamma_{2, i}\right)\right]\left[1-T_{0} \cdot J\left(\gamma_{1, i}\right)\right] \cdot I_{i},
\end{aligned}
$$

where $i$ is the number of the information channel; $i+4$ is the number of the mode that is emitted by the information channel of the system.

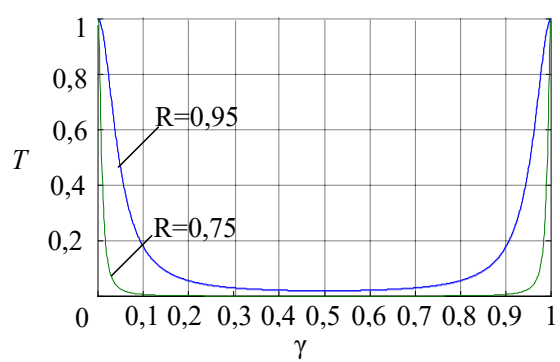

Fig. 6. The dependence of the FPI relative transmittance on $\gamma$ for various $R$

Thus, the obtained expressions for the initial LR of the MLMS channels show that the intensity of the selected modes decreases in each subsequent channel, overlapping the initial non-uniformity of the LR spectral components. Further amplification of the ACP selected spectral compo- 
nents in each individual MLMS channel makes it possible to eliminate this unevenness, as well as to provide the necessary radiation power of the aircraft, depending on the purpose and performance characteristics of the LIMS.

\section{Discussion of the results of the formation of multi-frequency signals of a laser informa- tion-measuring system}

The multifunctionality of proposed LIMS consists in the use of laser and optoelectronic modules (LM and OEM). In this case, the LM consists of measuring and information channels, and the OEM consists of television and infrared channels.

The selection of longitudinal modes from the spectrum of a single-mode multi-frequency with synchronization of longitudinal modes of LR is the basis for creating their combinations as a carrier frequency. This allows the channel to select probing signals on the transmitting side, which, after reflection from the aircraft, on the receiving side turn into the radio engineering range. Such signals are qualitatively distributed, amplified, and filtered to process the received measurement information about the aircraft MP.

Thanks to such signals, LIMS is an information-measuring complex. So, due to the interaction of channels, the conversion of carrier frequencies into intermodal beats of the radio range (intergeneric beat frequency), it gains new opportunities to increase the speed of information transfer and the accuracy of simultaneous measurements of aircraft MP. The proposed LIMS improves geodetic reference, allows the use of new types of digital meters and the like.

Narrow-band filtering is especially advantageous for measuring channels, due to the narrowing of the signal band significantly increase the accuracy of measuring aircraft MP.

The proposed theoretical foundations of the formation of laser multi-frequency signals for combining information and measurement channels of LIMS can simplify the construction of the structure of a multifunctional system. This makes the channels of the system independent (without mutual influence), since the dynamic range of the photodetector and the protective PB in frequency are sufficient in terms of signal level.

To create single-mode multi-frequency laser radiation, it is proposed to use either a solid-state laser pumped by laser diodes (which are characterized by small dimensions, high efficiency and good radiation coherence), or a semiconductor laser.

To synchronize the longitudinal modes of single-mode multi-frequency laser radiation, it is proposed to use the amplitude method of active synchronization of longitudinal modes using an acoustic-optical modulator. This will provide a stable mode of longitudinal mode synchronization in single-mode multi-frequency laser radiation and the necessary stabilization of the intermode beat frequencies.

It is advisable to direct further research in this direction to the modeling (synthesis) of measuring signals to determine the parameters of the movement of aircraft by laser information-measuring systems.

\section{Conclusions}

The list of initial data for the synthesis of measuring signals of laser information transmission systems is substantiated. It is proved that the spectrum of single-mode multi-frequency radiation has been formed, which allows one to distinguish combinations of longitudinal modes (frequencies) to create 4 partial radiation patterns of laser radiation. This justifies the possibility of using a single laser to transmit and receive information about the parameters of the aircraft movement.

The analytical basis for calculating the structure of signals at the output of a laser information-measuring system is proposed. It is analytically proven that the proposed device allows to transmit the electric fields of each frequency intermode beat in the radio range.

A modified longitudinal mode selector of a laser information-measuring system has been developed. Such a selector is proposed to be built on the basis of narrow-band Fabry-Perot interferometers. A functional diagram of the proposed selector is presented.

The calculations of the channel characteristics of the modified selector of longitudinal modes of the laser information-measuring system are done. The values of the maximum and minimum 
transmittance of the measuring channels are determined. The maximum transmittance during absorption of dielectric mirrors at all absorption values is more than 0.9. The minimum transmittance is between $1.7 \cdot 10^{-2}$ and $1.7 \cdot 10^{-5}$, depending on the reflection coefficient.

\section{References}

[1] Yoon, K. S., Jeong, E. J., Jeong, D. Y., Kang, C.-S. (2019). Development of a 3-Axis Displacement Measuring Heterodyne Interferometer System Usable with a He-Ne Laser of Either $3.76 \mathrm{MHz}$ or $20 \mathrm{MHz}$ Split Frequency. Proceedings of the 12th International Conference on Measurement and Quality Control - Cyber Physical Issue, 74-80. doi: https://oi.org/10.1007/9783-030-18177-2_7

[2] Han, Q. B., Shen, J. G., Jiang, X. P., Yin, C., Jia, J., Zhu, C. P. (2016). Propagation characteristic of laser-generated visco-elastic Rayleigh-like waves in stratified half-space. EURASIP Journal on Wireless Communications and Networking, 2016 (1). doi: https://doi.org/10.1186/s13638-016-0599-z

[3] Kazakov, E. L., Kazakov, A. E., Kolomiytsev, A. V.; Kazakov, E. L. (Ed.) (2015). Raspoznavanie tseley po signal'noy informatsii v odnopozitsionnyh i mnogopozitsionnyh lokatorah. Kharkiv: Miskdruk, 459.

[4] Zyryanov, Yu. T., Belousov, O. A., Fedyunin, P. A. (2011). Osnovy radiotehnicheskih sistem. Tambov: Izd-vo TGTU, 144.

[5] Aleshin, G. V., Bogdanov, Yu. A. (2008). Effektivnost' slozhnyh radio-tehnicheskih sistem. Kyiv: Naukova dumka, 288.

[6] Hu, Y. (2017). The Target Detection System Based on Laser Imaging. Theory and Technology of Laser Imaging Based Target Detection, 65-104. doi: https://doi.org/10.1007/978-981-10-3497-8_3

[7] Herasimov, S., Belevshchuk, Y., Ryapolov, I., Tymochko, O., Pavlenko, M., Dmitriiev, O. et. al. (2018). Characteristics of radiolocation scattering of the Su25T attack aircraft model at different wavelength ranges. Eastern-European Journal of Enterprise Technologies, 6 (9 (96)), 22-29. doi: https://doi.org/10.15587/1729-4061.2018.152740

[8] Basov, V. G. (2013). Izmeritel'nye signaly i funktsional'nye ustroystva ih obrabotki. Ch. 1: Izmeritel'nye signaly. Minsk: BGUIR, 119.

[9] Kudriashov, V. (2016). Experimental Evaluation of Opportunity to Improve the Resolution of the Acoustic Maps. Intelligent Systems Reference Library, 353-373. doi: https://doi.org/10.1007/978-3-319-32192-9_11

[10] Kozintsev, V. I., Belov, M. L., Orlov, V. M. (2006). Osnovy impul'snoy lazernoy lokatsii. Moscow: Izd-vo MGTU im. N. E. Baumana, 512.

[11] Marchenko, A. L., Marchenko, E. A. (2010). Osnovy preobrazovaniya informatsionnyh signalov. Moscow: Goryachaya liniya - Telekom, 286.

[12] Kiraci, E., Franciosa, P., Turley, G. A., Olifent, A., Attridge, A., Williams, M. A. (2016). Moving towards in-line metrology: evaluation of a Laser Radar system for in-line dimensional inspection for automotive assembly systems. The International Journal of Advanced Manufacturing Technology, 91 (1-4), 69-78. doi: https://doi.org/10.1007/s00170-016-9696-8

[13] Gruza, M., Gąska, P., Harmatys, W., Gąska, A. (2019). Assessment of Influence of Scanning Parameters on Uncertainty of Measurements Performed Using Laser Tracking System. Proceedings of the 12th International Conference on Measurement and Quality Control - Cyber Physical Issue, 201-208. doi: https://doi.org/10.1007/978-3-030-18177-2_19

[14] Ren, H., Yan, W. (2011). The Test Principle and Algorithm of Laser Measuring Tree Height. Lecture Notes in Electrical Engineering, 381-387. doi: https://doi.org/10.1007/978-3-642-25899-2_51

[15] Facas Vicente, M. A. (2013). Design, Modelation and Numerical Simulation of a Novel Artefact for Coordinate Measuring Machines Calibration Based on Laser Trilateration. Arabian Journal for Science and Engineering, 38 (6), 1519-1528. doi: https:// doi.org/10.1007/s13369-013-0537-x

[16] Vukašinović, N., Duhovnik, J. (2018). Optical 3D Geometry Measurments Based on Laser Triangulation. Advanced CAD Modeling, 191-216. doi: https://doi.org/10.1007/978-3-030-02399-7_9

[17] Sirotskiy, A. A. (2013). Proektirovanie pozitsionnyh lazernyh izmeritel'nyh sistem. Moscow: Sputnik +, 145.

[18] Ayswarya, P. R., Pournami, S. S., Ravi, N. (2015). A Survey on Ring Laser Gyroscope Technology. International Journal of Computer Applications, 116 (2), 25-27. doi: https://doi.org/10.5120/20310-2354

[19] Mordasov, V. I., Sazonnikova, N. A. (2006). Proektirovanie lazernyh sistem tehnologicheskih ustanovok. Samara: Izd-vo Samar. gos. aehrokosm. un-ta, 168.

[20] Herasimov, S., Pavlii, V., Tymoshchuk, O., Yakovlev, M. Y., Khaustov, D. Y., Ryzhov, Y. et. al. (2019). Testing Signals for Electronics: Criteria for Synthesis. Journal of Electronic Testing, 35 (3), 349-357. doi: https://doi.org/10.1007/s10836-019-05798-9 